Check for updates

Cite this: RSC Adv., 2021, 11, 5003

Received 30th November 2020

Accepted 21st January 2021

DOI: $10.1039 / \mathrm{dOra10094c}$

rsc.li/rsc-advances

\title{
Laser synthesis: a solvent-free approach for the preparation of phenylthiazolo[5,4-b]pyridine derivatives $\uparrow$
}

\author{
Rihab Jemili, ab Joana F. Campos, (D) a Nicolas Dumuis, ${ }^{b}$ Hervé Rabat, ${ }^{b}$ Nadjib Semmar ${ }^{b}$ \\ and Sabine Berteina-Raboin (D) *a
}

We describe here a rapid and straightforward solvent-free method to access phenylthiazolo[5,4- $b]$ pyridines using a Nd-YAG laser NANO-NY81-10 ( $\lambda=355 \mathrm{~nm}, 10 \mathrm{~Hz}$ pulse frequency; $8 \mathrm{~ns}$ pulse duration). This newly presented method successfully brings several improvements to the laser assisted synthesis of N,Sheterocycles. We are able to provide a solvent-, metal- and base-free method with good yield and a substantial reduction in reaction time.

\section{Introduction}

The thiazole heterocycle occupies a significant place in medicinal chemistry. This skeleton is present in various products with essential medicinal properties such as vitamin B1 (thiamine). This ring is also present in various products synthesized in the laboratory.

The preparation of thiazoles has soared in recent years, notably benzothiazole, which has proven its potential in successful biological applications (Fig. 1). Scott and Njardarson, from their analysis of sulfur-containing US FDA-approved drugs, pointed out that 23 of the approved drugs contain at least one thiazole and that 3 drugs contain a benzothiazole. Earlier, in 2014, Njardarson's team demonstrated that 4 of the 10 most commonly used nitrogen heterocycles also contain a sulfur atom and that thiazole is one of them. Additionally, they reported a remarkable fact: $67 \%$ of all thiazole-containing pharmaceuticals belong to the large class of $\beta$-lactam antibiotics. ${ }^{1-3}$ Photoreaction in organic synthesis is a domain that has been growing in recent years. Due to an increasing exploration and expansion effort, several teams have been involved in the construction of heterocycles in order to perform pharmacomodulation through another synthesis strategy, while obtaining derivatives with potential biological activity. ${ }^{4-8}$ We surmised that the use of laser energy could significantly improve the energy consumption of reactions compared to the activation of

${ }^{a}$ Institut de Chimie Organique et Analytique (ICOA), Université d'Orléans, UMR, CNRS 7311, BP 6759, rue de Chartres, 45067 Orléans cedex 2, France. E-mail: sabine. berteina-raboin@univ-orleans.fr

${ }^{b}$ GREMI (Groupe de Recherches sur l'Energétique des Milieux Ionisés), UMR CNRS 7344, Université d'Orléans, 14 rue d'Issoudun, BP 6744, 45067 Orléans Cedex 2, France

$\dagger$ Electronic supplementary information (ESI) available. See DOI: 10.1039/d0ra10094c media in conventional heating and could also lead to different compounds depending on the type of reaction envisaged. For this reason, in this work, our team reports an innovative and unexplored approach to the synthesis of $S, N$-heterocycles. It involves making use of a laser beam to obtain phenylthiazolo $[5,4-b]$ pyridine derivatives in order to develop the initial parameters since laser energy has seldom been studied in organic synthesis. Indeed, to the best of our knowledge, the construction of 9-azafluorene from 2-azidobiphenyl using LED, ${ }^{9}$ $\operatorname{laser}^{\mathbf{1 0}}$ (Table 1) or visible light ${ }^{\mathbf{1 1}}$ is the only reported preparation of nitrogen containing heterocycles in the literature.

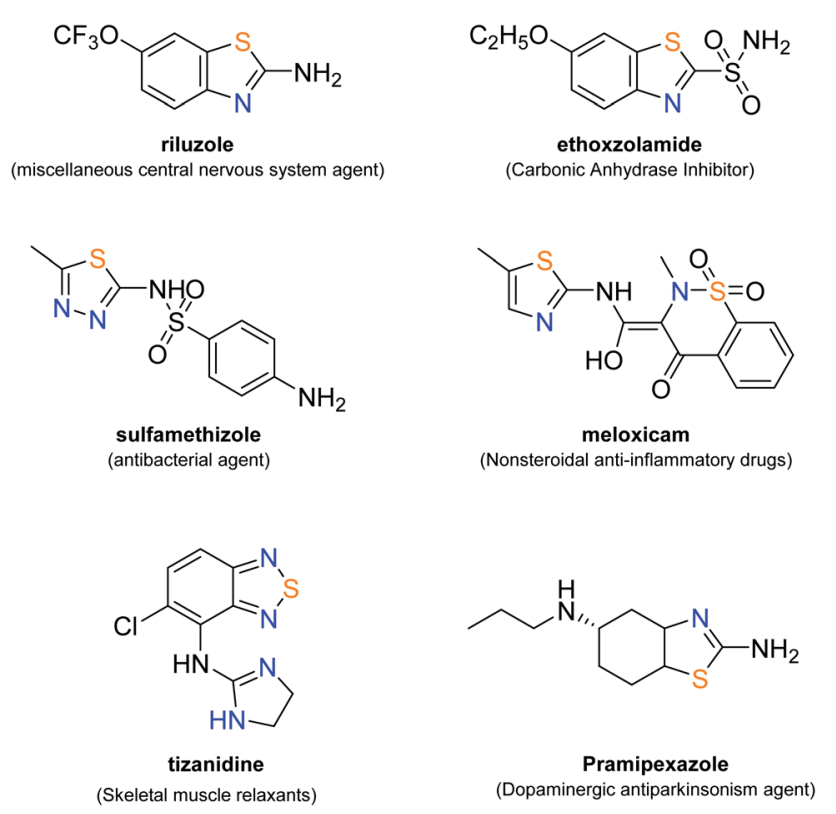

Fig. 1 Structure of some FDA approved drugs with skeletons similar to those reported in this article. 
Table 1 Comparison of equipment used in the study

\begin{tabular}{llll}
\hline Apparatus & Pulse frequency & Pulse duration & Ref. \\
\hline Nd-YAG laser & $8 \mathrm{kHz}$ & $26 \mathrm{~ns}$ & 10 \\
& $1-10 \mathrm{~Hz}$ & $10 \mathrm{~ns}$ & This work
\end{tabular}

Considering the above, it seemed important to explore this synthesis approach in order to improve general knowledge in the field of new and greener synthetic strategies for the construction of $O, S, N$-heterocycles. ${ }^{12-21}$ To prepare the benzothiazole skeleton, and after reviewing the literature, we found that Ramakrishnan's team had successfully used the potential of photochemical synthesis. The photocyclization of halogenated thioacetanilides gave a high yield of halogenated 2methylbenzothiazoles (Scheme 1a). ${ }^{22}$

The 2-(indol-1-yl)benzothiazoles were obtained in moderate to good yield from the irradiation of $\mathrm{N}$-phenyl-1 $\mathrm{H}$-indole-1carbothioamides at $254 \mathrm{~nm}$ for 8-55 h (Scheme 1b); ${ }^{23}$ 2-benzoylaminonaphtho[2,1- $d]$ thiazole was obtained from $N$-benzoyl$N^{\prime}$-(1-chloro-2-naphthyl)thiourea using $\mathrm{MeOH}$ as solvent for $12 \mathrm{~h}$ in $65 \%$ yield (Scheme 1c). ${ }^{24}$

A literature review to identify the best conditions for this cyclization from aminochloropyridines showed that the most commonly used conditions made use of EtOH and $\mathrm{MeOH}$ as solvent; the reaction time ranged from $16 \mathrm{~h}$ to $18 \mathrm{~h}$ using classical heating ${ }^{25-27}$ and $8 \mathrm{~h}$ to $55 \mathrm{~h}$ using photochemical irradiation. ${ }^{22-24}$

The use of laser energy allows a shorter reaction time, probably due to the energy being efficiently conducted by the beam which limits the energy loss that can occur either in conventional thermal heating or through the use of LED lamps for example.

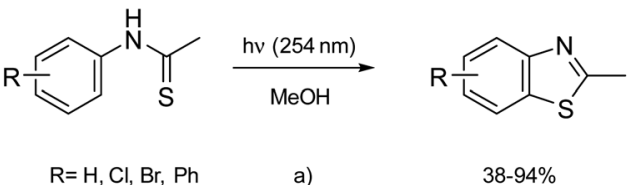

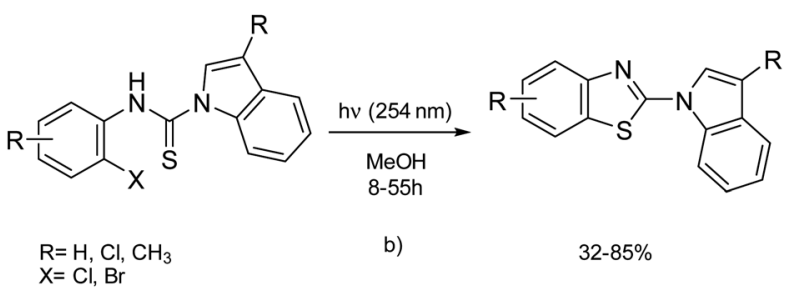<smiles>O=C(NC(=O)c1ccccc1)NC(=S)Nc1ccc2ccccc2c1Cl</smiles>

Scheme 1 Reported literature.

\section{Results and discussion}

To determine which laser was the most suitable for our purpose, and before starting the reaction optimization process, a UV spectrum of compound 1 was recorded.

The UV spectrum showed that 3-amino-2-chloropyridine has a maximum absorption at $311 \mathrm{~nm}$ in $\mathrm{EtOH}$, thus guiding us towards the most efficient reactant sensitization. From this information we determined the optic GAP of our starting material: $3.98 \mathrm{eV}$ at $311 \mathrm{~nm}$. Although the maximum absorption of 3-amino-2-chloropyridine in ethanol was at $311 \mathrm{~nm}$, it was decided to pursue this study at $355 \mathrm{~nm}$ as this starting compound presents very acceptable absorbance results at this wavelength; moreover, the laser available in our laboratory is at $355 \mathrm{~nm}$ and is not adjustable.

Photocyclisation optimization using an Nd-YAG laser NANONY81-10 ( $\lambda=355 \mathrm{~nm}, 10 \mathrm{~Hz}$ pulse frequency; pulse duration 8 ns) (Fig. 2 and 3) was carried out in two distinct parts. The influence of the solvent was studied first, then the amount of energy needed for the synthesis process was assessed.

Practically, we reacted 3-amino-2-chloropyridine (1 equiv.) with phenyl isothiocyanate ( 1 equiv.) and varied the amount of solvent or starting material (Fig. 4).

The purpose of this series of experiments was to demonstrate the influence of concentration on performance. In Fig. 4, two methods were tested $(2 \mathrm{~h}, 355 \mathrm{~nm}, 60 \mathrm{~mJ})$ : the first series consisted in keeping the amount of solvent constant $(1 \mathrm{~mL})$ and varying the amount of starting reactants to obtain the desired concentration (curve A). It can be seen that the yield improved with the reactant concentration. The second method (curve B) consisted in keeping the quantities of the starting products

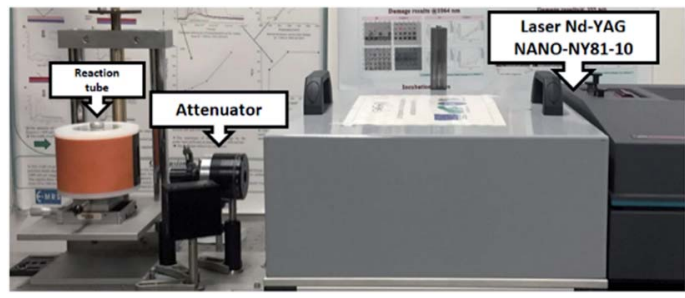

Fig. 2 Experimental setup with Nd-YAG laser NANO-NY81-10.

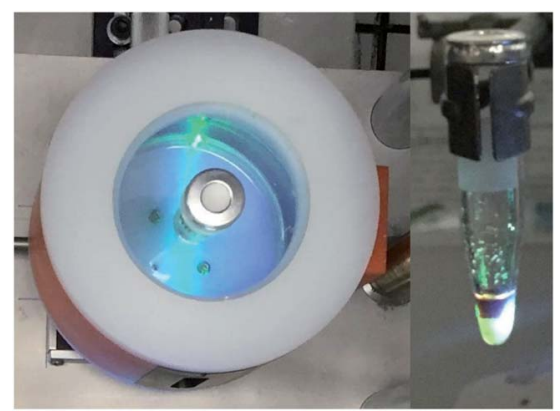

Fig. 3 Top view of photoreactor with reaction tube (left) and reaction tube (right). 


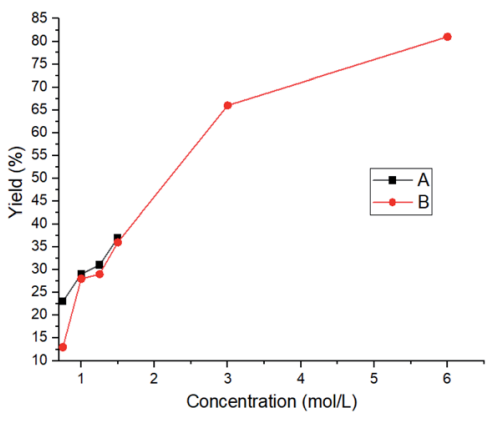

Fig. 4 Influence of the solvent on reaction yield.

constant and varying the quantity of solvent ( $2 \mathrm{~mL}, 1.5 \mathrm{~mL}, 1.25$ $\mathrm{mL}, 1 \mathrm{~mL}, 0.5 \mathrm{~mL}, 0.25 \mathrm{~mL})$. In this case, the solvent amount, hence the concentration of reactants, was limited due to the size of the reactor. The reactions were performed with peak power between 1.3 and 7.5 MW. As expected, the concentration allowed an increase in yield over the reaction time set at the start. In order to ensure an impact zone of the laser beam in relation to the reactor content, the quantities on which we worked were deliberately limited, although the reactors can contain a greater volume.

By observation, it was found that the absence of solvent was beneficial in this process and from these data, we moved on to phase 2 of the optimization, the energy study (Table 2).

The desired compound was obtained with an interesting yield range. Each time the energy input was decreased, the yield perceptibly worsened and the reaction time increased (Table 2, entry 1-6). This process proved to be a good alternative to the most commonly used synthetic routes. Based on our results, the scope and limitations of the optimized conditions for the preparation of phenylthiazolo[5,4- $b]$ pyridines were assessed using several isothiocyanates (Fig. 5). Concerning compounds 2 and 6, which could not be obtained, it is possible that the reactivity of the 3-pyridylisothiocyanate was not great enough and that there was a $\pi$ stacking problem with biphenylisothiocyanate.

Table 2 Influence of the energy input on reaction yield

\begin{tabular}{llll}
\hline & & & \\
& & & Yield $^{a}(\%)$ \\
\hline & & $(\mathrm{h})$ & Solvent free \\
Entry & Energy $^{b}(\mathrm{~mJ})$ & 2 & 90 \\
1 & $60 \pm 0.5$ & 3 & 80 \\
2 & $50 \pm 0.5$ & 4 & 72 \\
3 & $40 \pm 0.5$ & 4 & 55 \\
4 & $30 \pm 0.5$ & 4 & 15 \\
5 & $20 \pm 0.5$ & 4 & 8 \\
6 & $11 \pm 0.5$ & &
\end{tabular}

${ }^{a}$ Isolated yield after purification via column chromatography. ${ }^{b}$ Photon energy: $3.49 \mathrm{eV}$.
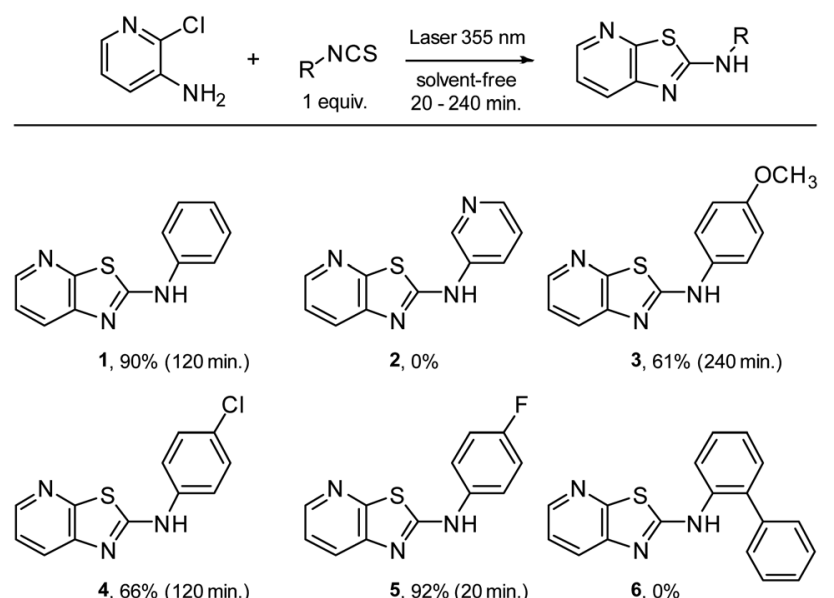

5, $92 \%(20 \mathrm{~min}$.
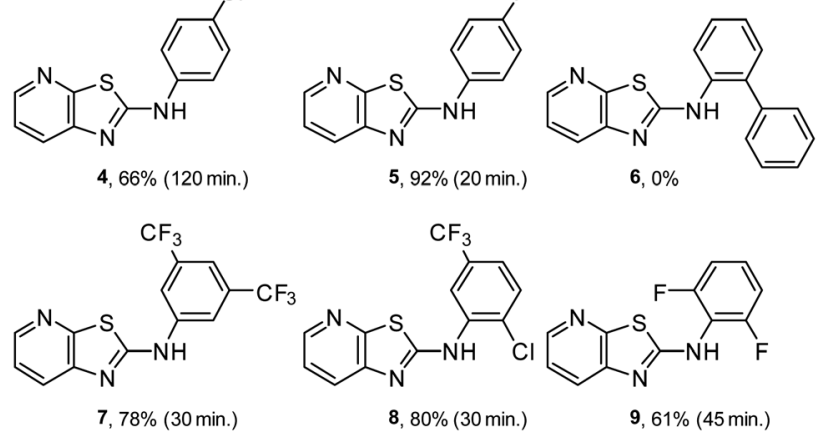

Fig. 5 Synthesis of phenylthiazolo[5,4-b]pyridines.

The phenylthiazolo[5,4- $b]$ pyridines 1-9 were synthesized in moderate to excellent yields, demonstrating the generalizability of this method (Fig. 5). The methodology presented successfully brings several improvements to the synthesis of $N, S$-heterocycles. Using laser synthesis, we are able to provide a solvent-, metal- and base-free methodology with good yield and a substantial reduction in reaction time.

\section{Experimental}

\section{Materials and methods: laser}

An Nd:YAG laser source was employed in this study. This laser source works at a wavelength of $1064 \mathrm{~nm}$ in the fundamental state. Thanks to the second and third harmonic generators, the 532 and $355 \mathrm{~nm}$ wavelengths are also available. The laser frequency (repetition rate) is also tunable from 1 to $10 \mathrm{~Hz}$, with a mean pulse time of $10 \mathrm{~ns}$. In the present experiments and due to the absorption coefficient of the glass employed (reaction tube), the maximum photon energy that could be used was $355 \mathrm{~nm}$. For this wavelength, the maximum output energy is 100

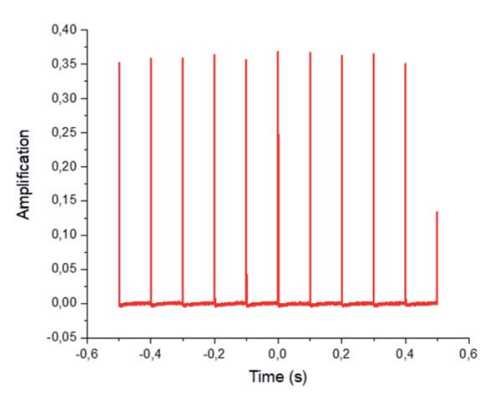

Fig. 6 Laser frequency. 


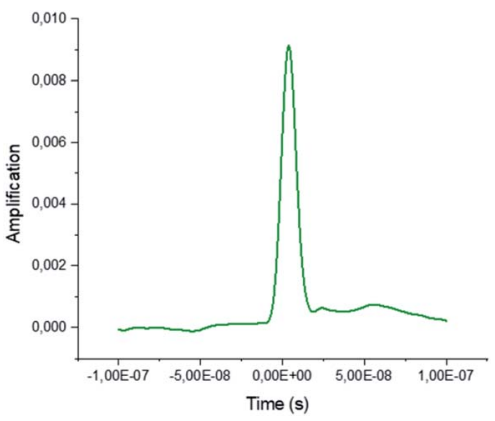

Fig. 7 Pulse duration of an Nd-YAG NANO-NY81-10 laser.

$\mathrm{mJ}$ per pulse. An optical polarizer device was also used to monitor the irradiation energy in the range 10 to $100 \mathrm{~mJ}$. A Gentec ${ }^{\circ}$ Joulemeter/Powermeter was employed for the directed measurement of the incident beam energy. The working conditions were $10 \mathrm{~Hz}$ for the repetition rate, with a pulse duration close to $10 \mathrm{~ns}$, measured thanks to a Hamamatsu ${ }^{\circledR}$ high rise time photodiode $(<500$ ps $)$ as shown in Fig. 6 and 7.

The tube holder/reactor is a cylindrical chamber that contains a metallic reflector on the wall position. The reactor was designed in CAD (Fig. 8) and the entire product construction was then carried out by 3D printing (stereo-lithography). Its internal diameter is $87 \mathrm{~mm}$ and its height $80 \mathrm{~mm}$. The reflecting wall is made of $0.5 \mathrm{~mm}$ thick polished stainless steel. All the other elements were produced using traditional machining.

As demonstrated using TracePro ${ }^{\circledR}$ software (Fig. 9: (A) schematic view of the incident beam interaction with the glass tube; (B) TracePro® simulation of the multireflection inside the reactor), this reflector helps to ensure that all reflected parts of the incident laser beam are finally focused into the tube center. Thanks to an $X, Y, Z$ micro-positioning stage, the irradiated zone on the tube is also fully controlled.

\section{Synthetic procedures}

General procedure. In a sealed tube, the 3-amino-2chloropyridine $(200 \mathrm{mg}, 1.55 \mathrm{mmol})$ was mixed with the isothiocyanate (1 eq.). The tube was irradiated with an Nd-YAG nanosecond laser $(\lambda=355 \mathrm{~nm})$. The product thus formed was purified with a chromatography column, $\mathrm{CH}_{2} \mathrm{Cl}_{2} / \mathrm{MeOH}$ $(90: 10)$.

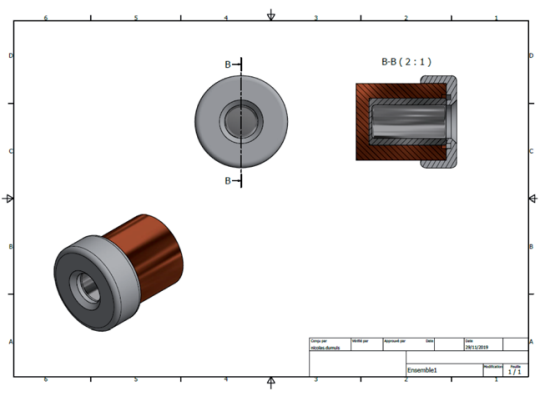

Fig. 8 3D representation of the reactor.

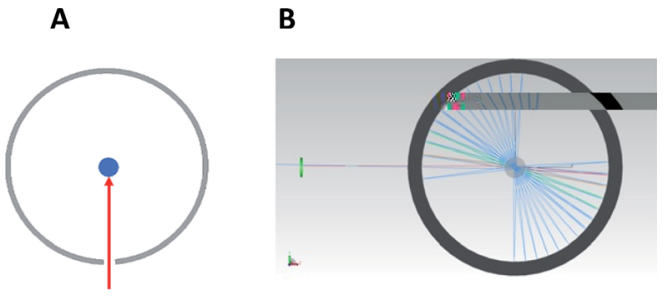

Fig. 9 (A) represents schematic view of the incident beam interaction with the glass tube; (B) represents TracePro ${ }^{\circledR}$ simulation of the multireflection inside the reactor.

\section{Conclusions}

The use of a new, simple and efficient solvent-free procedure enabled the preparation of phenylthiazolo[5,4-b]pyridine derivatives. This laser synthesis protocol proved to be a good alternative to the most commonly used synthetic routes. In this study, in addition to eliminating the need for solvent, two reaction parameters were improved: time and yield. These first encouraging results will be the basis of our future studies on the use of laser energy applied to the synthesis of interesting organic compounds and on the study of reaction mechanisms.

\section{Author contributions}

J. F. C. and S. B. R. conceived and designed the synthetic pathway. R. J. and N. S. were involved in planning the laser synthesis. N. D. built the reactor. H. R. was responsible for the maintenance of the laser. R. J. performed the experiments. J. F. C. collected the data and drafted the initial version of the manuscript. S. B. R. and N. S. reviewed and edited the manuscript.

\section{Conflicts of interest}

There are no conflicts to declare.

\section{Acknowledgements}

This work is part of the project entitled "APR-IR SPLACh" funded by the Region Centre-Val-de-Loire.

\section{References}

1 K. A. Scott and J. T. Njardarson, Analysis of US FDA-Approved Drugs Containing Sulfur Atoms, Top. Curr. Chem., 2018, 376, 5, DOI: 10.1007/s41061-018-0184-5.

2 M. T. Chhabria, S. Patel, P. Modi and P. S. Brahmkshatriya, Thiazole: A Review on Chemistry, Synthesis and Therapeutic Importance of its Derivatives, Curr. Top. Med. Chem., 2016, 16, 2841-2862, DOI: 10.2174/ 1568026616666160506130731.

3 E. Vitaku, D. T. Smith and J. T. Njardarson, Analysis of the structural diversity, substitution patterns, and frequency of nitrogen heterocycles among U.S. FDA approved 
pharmaceuticals, J. Med. Chem., 2014, 57, 10257-10274, DOI: 10.1021/jm501100b.

4 N. Kaur, P. Bhardwaj, M. Devi, Y. Verma and P. Grewal, Photochemical reactions in five and six-membered polyheterocycles synthesis, Synth. Commun., 2019, 49, 2281-2318, DOI: 10.1080/00397911.2019.1622732.

5 N. Kaur, Photochemical reactions as key steps in fivemembered $N$-heterocycle synthesis, Synth. Commun., 2018, 48, 1259-1284, DOI: 10.1080/00397911.2018.1443218.

6 N. Kaur, Synthesis of seven and higher membered nitrogen containing heterocycles using photochemical irradiation, Synth. Commun., 2018, 48, 2815-2849, DOI: 10.1080/ 00397911.2018 .1501488$.

7 N. Kaur, Photochemical Reactions: Synthesis of Sixmembered $N$-heterocycles, Curr. Org. Chem., 2017, 14, 972998, DOI: $10.2174 / 1570179414666170201150701$.

8 K. Mizuno, Y. Nishiyama, T. Ogaki, K. Terao, H. Ikeda and K. Kakiuchi, Utilization of microflow reactors to carry out synthetically useful organic photochemical reactions, $J$. Photochem. Photobiol., C, 2016, 29, 107-147, DOI: 10.1016/ j.jphotochemrev.2016.10.002.

9 S. Parisien-Colette, C. Cruché, X. Abel-Snape and S. K. Collins, Photochemical intramolecular amination for the synthesis of heterocycles, Green Chem., 2017, 19, 47984803, DOI: 10.1039/c7gc02261a.

10 E. Bremus-Köbberling, A. Gillner, F. Avemaria, C. Réthoré and S. Bräse, Photochemistry with laser radiation in condensed phase using miniaturized photoreactors, Beilstein J. Org. Chem., 2012, 8, 1213-1218, DOI: 10.3762/ bjoc.8.135.

11 L. Yang, Y. Zhang, X. Zou, H. Lu and G. Li, Visible-lightpromoted intramolecular $\mathrm{C}-\mathrm{H}$ amination in aqueous solution: synthesis of carbazoles, Green Chem., 2018, 20, 1362-1366, DOI: 10.1039/c7gc03392c.

12 J. F. Campos, A. Pacheco-Benichou, C. Fruit, T. Besson and S. Berteina-Raboin, Synthesis of Benzo-Fused $11 H$-Pyrido [2,1-b]quinazolin-11-ones by a Buchwald-Hartwig Coupling/Pyridine Dearomatization Sequence in Eucalyptol, Synthesis, 2020, 52(20), 3071-3076, DOI: 10.1055/s-0040-1707158.

13 J. F. Campos, M. Loubidi, M.-C. Scherrmann and S. BerteinaRaboin, A Greener and Efficient Method for Nucleophilic Aromatic Substitution of Nitrogen-Containing Fused Heterocycles, Molecules, 2018, 23, 684, DOI: 10.3390/ molecules23030684.

14 J. F. Campos, M.-C. Scherrmann and S. Berteina-Raboin, Eucalyptol: a new solvent for the synthesis of heterocycles containing oxygen, sulfur and nitrogen, Green Chem., 2019, 21, 1531-1539, DOI: 10.1039/c8gc04016h.

15 J. F. Campos and S. Berteina-Raboin, Eucalyptol as a BioBased Solvent for Buchwald-Hartwig Reaction on $\mathrm{O}, \mathrm{S}, \mathrm{N}$ Heterocycles, Catalysts, 2019, 9, 840-850, DOI: 10.3390/ catal9100840.
16 J. F. Campos and S. Berteina-Raboin, Eucalyptol as bio-based solvent for Migita-Kosugi-Stille coupling reaction on $\mathrm{O}, \mathrm{S}, \mathrm{N}$ heterocycle, Catal. Today, 2020, 358, 138-142, DOI: 10.1016/ j.cattod.2019.11.004.

17 N. Fresneau, M.-A. Hiebel, L. A. Agrofoglio and S. BerteinaRaboin, Efficient Synthesis of Unprotected C-5-Aryl/ Heteroaryl-2'-deoxyuridine via a Suzuki-Miyaura Reaction in Aqueous Media, Molecules, 2012, 17, 14409-14417, DOI: 10.3390/molecules171214409.

18 N. Fresneau, M.-A. Hiebel, L. A. Agrofoglio and S. BerteinaRaboin, One-pot Sonogashira-cyclization protocol to obtain substituted furopyrimidine nucleosides in aqueous conditions, Tetrahedron Lett., 2012, 53, 1760-1763, DOI: 10.1016/j.tetlet.2012.01.106.

19 M.-A. Hiebel, Y. Fall, M.-C. Scherrmann and S. BerteinaRaboin, Straightforward Synthesis of Various 2,3-Diarylimidazo[1,2- $a$ ]pyridines in $\mathrm{PEG}_{400}$ Medium through One-Pot Condensation and $\mathrm{C}-\mathrm{H}$ Arylation, Eur. J. Org. Chem., 2014, 21, 4643-4650, DOI: 10.1002/ ejoc. 201402079.

20 M.-A. Hiebel and S. Berteina-Raboin, Iodine-catalyzed regioselective sulfenylation of imidazoheterocycles in PEG $_{400}$, Green Chem., 2015, 17, 937-944, DOI: 10.1039/ c4gc01462f.

21 G. Dumonteil, M.-A. Hiebel, M.-C. Scherrmann and S. Berteina-Raboin, Iodine-catalyzed formation of substituted 2-aminobenzothiazole derivatives in $\mathrm{PEG}_{400}$, RSC Adv., 2016, 6, 73517-73521, DOI: 10.1039/c6ra15971k.

22 R. Paramasivam, R. Palaniappan and V. T. Ramakrishnan, Photochemical synthesis of 2-methylbenzothiazoles, $J$. Chem. Soc., Chem. Commun., 1979, 260-261, DOI: 10.1039/ c39790000260.

23 S. Muthusamy, R. Paramasivam and V. T. Ramakrishnan, Photochemical synthesis of 2-substituted benzothiazoles, $J$. Heterocycl. Chem., 1991, 28, 759-763, DOI: 10.1002/ jhet.5570280335.

24 G. Jayanthi, S. Muthusamy and V. T. Ramakrishnan, Photoreaction of $N$-arylcarbonyl- $N$-arylthiourea derivatives, J. Photochem. Photobiol., C, 1998, 116, 103-108, DOI: 10.1016/s1010-6030(98)00294-9.

25 H. G. Choi, J. Zhang, E. Weisberg, J. D. Griffin, T. Sim and N. S. Gray, Development of 'DFG-out' inhibitors of gatekeeper mutant kinases, Bioorg. Med. Chem. Lett., 2012, 22, 5297-5302, DOI: 10.1016/j.bmcl.2012.06.036.

$26 \mathrm{H}$. W. Aitland and G. A. Molander, A facile synthesis of 2-aminothiazolo[5,4-b]- and 2-aminothiazolo[4,5-c] pyridines, J. Heterocycl. Chem., 1977, 14, 129-134, DOI: 10.1002/jhet.5570140125.

$27 \mathrm{H}$. W. Altlang, The Smiles rearrangement of 2-tetrazolylthio3-aminopyridines, J. Org. Chem., 1976, 41, 3395-3399, DOI: 10.1021/jo00883a013. 